\title{
Robust Synchronization of A Class of Coupled Delayed Networks with Multiple Stochastic Disturbances: the Continuous-Time Case
}

\author{
Jinling Liang ${ }^{a, *}$, Zidong Wang ${ }^{b}$ and Xiaohui Liu $^{b}$
}

\begin{abstract}
In this paper, the robust synchronization problem is investigated for a new class of continuous-time complex networks that involve parameter uncertainties, time-varying delays, constant and delayed couplings, as well as multiple stochastic disturbances. The norm-bounded uncertainties exist in all the network parameters after decoupling, and the stochastic disturbances are assumed to be Brownian motions that act on the constant coupling term, the delayed coupling term as well as the overall network dynamics. Such multiple stochastic disturbances could reflect more realistic dynamical behaviors of the coupled complex network presented within a noisy environment. By using a combination of the Lyapunov functional method, the robust analysis tool, the stochastic analysis techniques and the properties of Kronecker product, we derive several delay-dependent sufficient conditions that ensure the coupled complex network to be globally robustly synchronized in the mean square for all admissible parameter uncertainties. The criteria obtained in this paper are in the form of linear matrix inequalities (LMIs) whose solution can be easily calculated by using the standard numerical software. The main results are shown to be general enough to cover many existing ones reported in the literature. Simulation examples are presented to demonstrate the feasibility and applicability of the proposed results.
\end{abstract}

Keywords

Robust synchronization; coupled complex networks; stochastic disturbance; Lyapunov functional; Kronecker product; time-varying delay; linear matrix inequality.

\section{INTRODUCTION}

Many systems in nature can be modelled as complex networks. The examples include, but are not limited to, neuronal networks, genetic networks, networks in social sciences, power grid networks, coupled mechanical systems, information networks and others. Complex networks pose new challenges for the understanding of cluster formation, stability, sensitivity and robustness, bifurcations and chaos, etc. The connection weights of the nodes of complex networks are largely dependent on certain resistance and capacitance values that include uncertainties (modelling errors). Also, the signal transfer could be perturbed randomly from the release of probabilistic causes such as neurotransmitters [21,34] and packet dropouts [35]. When analyzing the dynamical behaviors of complex networks, stochastic disturbances and modelling errors are probably two of the main sources that result in uncertainties. Therefore, robust dynamics analysis for uncertain stochastic complex networks has emerged as a challenging research issue. Note that the term 'robust' would appear when discussing a property of systems/networks where parameter uncertainties exist [36-40].

Among many others, synchronization is one of the most important dynamical behaviors for complex networks and has therefore received recurrent research interests in recent years. For example, the synchronization

This work was supported in part by the Royal Society of U.K., the National Natural Science Foundation of China under Grant 60804028 and Grant 61028008, the Teaching and Research Fund for Excellent Young Teachers at Southeast University of China, the Qing Lan Project of Jiangsu Province of China, the International Science and Technology Cooperation Project of China under Grant 2009DFA32050, and the Alexander von Humboldt Foundation of Germany.

${ }^{a}$ Department of Mathematics, Southeast University, Nanjing 210096, P. R. China.

${ }^{b}$ Department of Information Systems and Computing, Brunel University, Uxbridge, Middlesex, UB8 3PH, United Kingdom. Email: Zidong.Wang@brunel.ac.uk

${ }^{*}$ Corresponding author. Email: jinlliang@gmail.com 
problem has been thoroughly investigated for the large-scale networks of chaotic oscillators [15, 27, 33], the coupled systems exhibiting spatio-temporal chaos and autowaves [29,46], and the array of coupled neural networks $[18,22,23,26,28,42]$. On the other hand, time delay is recognized as an ubiquitous phenomenon in networks due to the facts that: 1) the limited speed of signals travelling through the links [10,11]; 2) couplings are frequently delayed in biological neural networks, gene regulatory networks, communication networks and electrical power grids $[3,10,17,20]$; and 3) time delays can cause complex dynamics such as periodic or quasiperiodic motions, Hopf bifurcation and higher-dimensional chaos. Therefore, synchronization problems for various networks have recently been extensively studied, see e.g. $[3,7,10,13,14,17,20,24,25,31,32,42,44,47]$ and the references cited therein.

It is worth pointing out that, in most existing literature, the synchronization problem has been investigated mainly for deterministic and precise complex networks with or without delays, see e.g. $[3,5,10,18,24,41]$. For example, in [41], the dynamical behavior has been studied for an array of identical differential equations with linear coupling. Considering of the delay effects, the authors of [5] and [18] have dealt with the global synchronization problems, respectively, for a coupled delayed neural network and a complex network with coupling delays. Elegant results have been obtained in $[5,18]$ under the assumptions that 1) the outercoupling matrix of the networks is required to be irreducible so as to avoid isolated clusters; and 2) the inner-coupling matrix is restricted to be symmetric. Very recently, a great deal of research efforts have been made towards the improvement of the results in $[5,18]$ under milder assumptions $[2,4,10]$. In particular, the properties of Kronecker products have been utilized in [3] and some sufficient criteria have been acquired in the form of linear matrix inequalities (LMIs) that can be readily solved. In the few papers tackling the synchronization problem for stochastic networks [4,45], the stochastic coupling term has been introduced. However, the deterministic couplings as well as the overall stochastic disturbances have not been taken into account in [4]. Moreover, neither the coupling terms nor the parameter uncertainties have been considered in [45] where only two sub-systems (i.e., the master system and slave system) have been investigated. So far, to the best of the authors' knowledge, the robust synchronization problem has received very little research attention for complex networks in the simultaneous presence of parameter uncertainties, time-varying delays, mixed couplings and multiple stochastic disturbances. Such a situation motivates our present investigation.

In this paper, we aim to challenge the robust synchronization problem for a new class of continuoustime complex networks with parameter uncertainties, time-varying delays, constant and delayed couplings, as well as multiple stochastic disturbances. The norm-bounded uncertainties enter into all the network state matrices, and the multiple stochastic disturbances influence the constant coupling term, the delayed coupling term as well as the overall network dynamics. Note that the considered multiple stochastic disturbances could better describe the dynamical behavior of a coupled complex network presented within a noisy environment. With the help from the Lyapunov functional method and the properties of Kronecker product, we employ the robust analysis tool and the stochastic analysis techniques to derive several delay-dependent sufficient conditions under which the coupled complex network is globally robustly synchronized in the mean square for all admissible parameter uncertainties. The criteria obtained in this paper are in the form of LMIs whose solution can be easily calculated by using the standard numerical software. We show that our main results can cover many existing ones reported in the literature. Simulation examples are presented to illustrate the usefulness of our results.

The rest of the paper is organized as follows. In Section II, an uncertain stochastically coupled complex model with time-varying delays is proposed and some preliminaries are briefly outlined. In Section III, by utilizing the Lyapunov functional method, we conduct the robust and stochastic analysis to obtain some delay-dependent sufficient criteria in terms of LMIs, so as to ensure the considered complex network to be globally robustly synchronized in the mean square. In Section IV, simulation examples are provided to show the effectiveness of the obtained results and, finally, conclusions are drawn in Section V. 
Notations: Throughout this paper, $I_{m}$ is the $m$-dimensional identity matrix. $\mathbb{R}^{n}$ and $\mathbb{R}^{m \times n}$ are, respectively, the $n$-dimensional Euclidean space and the set of all $m \times n$ real matrices. $P>0$ (respectively, $P_{1}>P_{2}$ ) means that matrix $P$ (respectively, $P_{1}-P_{2}$ ) is real, symmetric and positive definite. The superscript " $T$ " stands for matrix transposition and the asterisk " $*$ " in a matrix is used to represent the term which is induced by symmetry. $\|\cdot\|$ refers to the Euclidean vector norm and its induced matrix norm. The Kronecker product of matrices $Q \in \mathbb{R}^{m \times n}$ and $R \in \mathbb{R}^{p \times q}$ is a matrix in $\mathbb{R}^{m p \times n q}$ and denoted as $Q \otimes R$. Let $(\Omega, \mathcal{F}, \mathcal{P})$ be a complete probability space with a filtration $\left\{\mathcal{F}_{t}\right\}_{t \geq 0}$ containing all $\mathcal{P}$-null sets and being right continuous. $L_{\mathcal{F}_{0}}^{p}$ denotes the family of all $\mathcal{F}_{0}$-measurable $C\left([-\tau, 0], \mathbb{R}^{n}\right)$-valued random variables $\varphi=\{\varphi(s) \mid-\tau \leq s \leq 0\}$ such that $\sup _{-\tau \leq s \leq 0} \mathbb{E}\left\{|\varphi(s)|^{p}\right\}<\infty$, where $\mathbb{E}\{\cdot\}$ is the mathematical expectation operator with respect to the given probability measure $\mathcal{P}$. Sometimes, when no confusion would arise, the dimensions of a function or a matrix will be omitted for convenience.

\section{Model FORMUlation AND PRELIMINARIES}

Consider the following array of identical uncertain complex networks with stochastic coupling and disturbances:

$$
\begin{aligned}
d x_{i}(t)= & {\left[(A+\triangle A) x_{i}(t)+(D+\triangle D) x_{i}(t-\tau(t))+(B+\triangle B) f\left(x_{i}(t)\right)\right] d t+\sigma_{i}\left(t, x_{i}(t), x_{i}(t-\tau(t))\right) d \omega_{3}(t) } \\
& +\sum_{j=1}^{N} G_{i j}^{(1)} \Gamma_{1} x_{j}(t)\left(d t+d \omega_{1}(t)\right)+\sum_{j=1}^{N} G_{i j}^{(2)} \Gamma_{2} x_{j}(t-\tau(t))\left(d t+d \omega_{2}(t)\right), \quad t>0
\end{aligned}
$$

where $i=1,2, \ldots, N$ and $N$ is the number of coupled subsystems; $x_{i}(t)=\left(x_{i 1}(t), x_{i 2}(t), \ldots, x_{i n}(t)\right)^{T} \in \mathbb{R}^{n}$ is the state vector of the $i$-th complex network; $A, D$ and $B$ are the known connection weight matrices; the parameter uncertainties $\Delta A, \Delta D$ and $\Delta B$ are of the following structure

$$
\left[\begin{array}{lll}
\Delta A & \Delta D & \Delta B
\end{array}\right]=\operatorname{EF}\left[\begin{array}{lll}
M_{1} & M_{2} & M_{3}
\end{array}\right]
$$

where $E, M_{1}, M_{2}$ and $M_{3}$ are known real constant matrices with appropriate dimensions and the uncertain matrix $\mathcal{F}$ is unknown but satisfies

$$
\mathcal{F}^{T} \mathcal{F} \leq I .
$$

The delay $\tau(t)$ is time-varying, bounded and differentiable with

$$
0 \leq \tau(t) \leq \tau_{M}, \quad \dot{\tau}(t) \leq \mu ; \quad \forall t \in \mathbb{R}^{+}
$$

where $\tau_{M} \geq 0$ and $\mu$ are known constants. Moreover, $\omega_{1}(t), \omega_{2}(t)$ and $\omega_{3}(t)$ are mutually independent scalar Brownian motions defined on $(\Omega, \mathcal{F}, \mathcal{P})$ satisfying

$$
\mathbb{E}\left\{d \omega_{i}(t)\right\}=0 \text { and } \mathbb{E}\left\{\left[d \omega_{i}(t)\right]^{2}\right\}=d t, \quad i=1,2,3 ; t \in \mathbb{R}^{+} .
$$

The noise intensity function vector $\sigma_{i}: \mathbb{R} \times \mathbb{R}^{n} \times \mathbb{R}^{n} \rightarrow \mathbb{R}^{n}$ satisfies the Lipschitz condition, i.e., there exist constant matrices $W_{1}$ and $W_{2}$ of appropriate dimensions such that the following inequality

$$
\left(\sigma_{i}\left(t, u_{1}, v_{1}\right)-\sigma_{j}\left(t, u_{2}, v_{2}\right)\right)^{T}\left(\sigma_{i}\left(t, u_{1}, v_{1}\right)-\sigma_{j}\left(t, u_{2}, v_{2}\right)\right) \leq\left\|W_{1}\left(u_{1}-u_{2}\right)\right\|^{2}+\left\|W_{2}\left(v_{1}-v_{2}\right)\right\|^{2}
$$

holds for all $t \in \mathbb{R}^{+} ; i, j=1,2, \ldots, N$ and $u_{1}, v_{1}, u_{2}, v_{2} \in \mathbb{R}^{n}$. Furthermore, $\Gamma_{1}$ and $\Gamma_{2}$ are matrices describing the inner-coupling between the subsystems at time $t$ and $t-\tau(t)$, respectively; $G^{(1)}=\left(G_{i j}^{(1)}\right)_{N \times N}$ and $G^{(2)}=\left(G_{i j}^{(2)}\right)_{N \times N}$ are the outer-coupling configuration matrices representing the coupling strength and the topological structure of the complex networks that satisfy the following conditions

$$
G_{i j}^{(k)}=G_{j i}^{(k)} \geq 0 \quad(j \neq i) \quad \text { and } \quad G_{i i}^{(k)}=-\sum_{j=1, j \neq i}^{N} G_{i j}^{(k)} ; \quad i, j=1,2, \ldots, N ; k=1,2 .
$$


Finally, $f(\cdot): \mathbb{R}^{n} \rightarrow \mathbb{R}^{m}$ is an unknown nonlinear function of the network and is assumed to satisfy sectorbounded condition given in Assumption 1.

Assumption 1: There exist real constant matrices $X_{1}$ and $X_{2} \in \mathbb{R}^{m \times n}$ such that

$$
\left(f(u)-f(v)-X_{1}(u-v)\right)^{T}\left(f(u)-f(v)-X_{2}(u-v)\right) \leq 0, \quad \forall u, v \in \mathbb{R}^{n}
$$

where $X_{2}-X_{1}$ is symmetric and positive definite.

Remark 1: The structure of the deterministic uncertainties as in (2)-(3) has been widely used in many papers of the problem of robust control and robust filtering, see e.g. [35]. On the other hand, the nonlinear function $f(\cdot)$ satisfying Assumption 1 is said to belong to the sector $\left[X_{1}, X_{2}\right]$ and, as pointed out in [16], the nonlinear description (8) is more general than the usually used Lipschitz conditions as in [3-5].

Remark 2: In the network model (1), stochastic disturbances are introduced on both the coupling term and the overall network because the network coupling could occur in both a deterministic and a stochastic way. Note that the stochastic coupling term was first proposed in [4] for investigating the complete synchronization problem of an array of linearly stochastically coupled neural networks with time delays, but the deterministic coupling as well as the overall stochastic disturbances have not been taken into account in [4]. Furthermore, the parameter uncertainties resulting mainly from measurement errors have been included in the model (1), which renders the model (1) even more general to reflect the reality.

The initial conditions associated with system (1) are given by

$$
x_{i}(s)=\varphi_{i}(s), \quad-\tau_{M} \leq s \leq 0, i=1,2, \ldots, N
$$

where $\varphi_{i}(\cdot) \in L_{\mathcal{F}_{0}}^{2}\left(\left[-\tau_{M}, 0\right], \mathbb{R}^{n}\right)$; and the corresponding state trajectory is denoted as $x_{i}\left(t, \varphi_{i}\right)$.

We are now ready to introduce the notion of robust synchronization for complex networks (1) with stochastic coupling and disturbances.

Definition 1: The coupled complex networks (1) is said to be globally robustly synchronized in the mean square if, for all admissible uncertainties $(2)-(3)$ and for all $\varphi_{i}(\cdot) \in L_{\mathcal{F}_{0}}^{2}\left(\left[-\tau_{M}, 0\right], \mathbb{R}^{n}\right)$, the following holds:

$$
\lim _{t \rightarrow \infty} \mathbb{E}\left\{\left\|x_{i}\left(t, \varphi_{i}\right)-x_{j}\left(t, \varphi_{j}\right)\right\|^{2}\right\}=0, \quad 1 \leq i<j \leq N
$$

By denoting

$$
\bar{A}=A+\Delta A, \quad \bar{D}=D+\Delta D, \quad \bar{B}=B+\Delta B
$$

and utilizing the Kronecker product $\otimes$, the network system (1) can be rewritten in a compact form as

$$
\begin{aligned}
d x(t)= & {\left[\left(I_{N} \otimes \bar{A}+G^{(1)} \otimes \Gamma_{1}\right) x(t)+\left(I_{N} \otimes \bar{D}+G^{(2)} \otimes \Gamma_{2}\right) x(t-\tau(t))+\left(I_{N} \otimes \bar{B}\right) F(x(t))\right] d t } \\
& +\sigma(t, x(t), x(t-\tau(t))) d \omega_{3}(t) \\
& +\left(G^{(1)} \otimes \Gamma_{1}\right) x(t) d \omega_{1}(t)+\left(G^{(2)} \otimes \Gamma_{2}\right) x(t-\tau(t)) d \omega_{2}(t), \quad t>0
\end{aligned}
$$

where $x(t)=\left(x_{1}^{T}(t), x_{2}^{T}(t), \ldots, x_{N}^{T}(t)\right)^{T}, F(x(t))=\left(f^{T}\left(x_{1}(t)\right), f^{T}\left(x_{2}(t)\right), \ldots, f^{T}\left(x_{N}(t)\right)\right)^{T}$ and $\sigma(t, x(t), x(t-$ $\tau(t)))=\left(\sigma_{1}^{T}\left(t, x_{1}(t), x_{1}(t-\tau(t))\right), \sigma_{2}^{T}\left(t, x_{2}(t), x_{2}(t-\tau(t))\right), \ldots, \sigma_{N}^{T}\left(t, x_{N}(t), x_{N}(t-\tau(t))\right)\right)^{T}$.

Before starting the main results, some lemmas needed in the next section are given as follows.

Lemma 1: [6] Let $\alpha \in \mathbb{R}$ and $X, Y, P, Q$ be matrices with appropriate dimensions. Then the following statements are true:

(1) $(\alpha X) \otimes Y=X \otimes(\alpha Y)$;

(2) $(X+Y) \otimes P=X \otimes P+Y \otimes P, P \otimes(X+Y)=P \otimes X+P \otimes Y$

(3) $(X \otimes Y)(P \otimes Q)=(X P) \otimes(Y Q)$;

(4) $(X \otimes Y)^{T}=X^{T} \otimes Y^{T}$. 
Lemma 2: [1] Let $Q(x)=Q^{T}(x), R(x)=R^{T}(x)$, and $S(x)$ depend affinely on $x$. Then the following linear matrix inequality

$$
\left[\begin{array}{cc}
Q(x) & S(x) \\
S^{T}(x) & R(x)
\end{array}\right]>0,
$$

holds if and only if one of the following conditions holds:

(1) $R(x)>0, Q(x)-S(x) R^{-1}(x) S^{T}(x)>0$;

(2) $Q(x)>0, R(x)-S^{T}(x) Q^{-1}(x) S(x)>0$.

Lemma 3: [12] (Jensen inequality) For any constant matrix $P \in \mathbb{R}^{n \times n}, P^{T}=P>0$, scalar $r>0$, and vector function $u:[0, r] \rightarrow \mathbb{R}^{n}$, one has

$$
r \int_{0}^{r} u^{T}(s) P u(s) d s \geq\left(\int_{0}^{r} u(s) d s\right)^{T} P\left(\int_{0}^{r} u(s) d s\right),
$$

provided that the integrals are well defined.

Lemma 4: Let $e$ be an $N$-dimensional vector with all components being 1 and $U=N I_{N}-e e^{T}$. For $i=1,2, \ldots, N$, assume that $P$ is an $n \times n$ matrix, $x=\left(x_{1}^{T}, x_{2}^{T}, \ldots, x_{N}^{T}\right)^{T}$ where $x_{i}=\left(x_{i 1}, x_{i 2}, \ldots, x_{i n}\right)^{T} \in \mathbb{R}^{n}$, and $y=\left(y_{1}^{T}, y_{2}^{T}, \ldots, y_{N}^{T}\right)^{T}$ where $y_{i}=\left(y_{i 1}, y_{i 2}, \ldots, y_{i n}\right)^{T} \in \mathbb{R}^{n}$. Then, we have the following relationships:

(1) $U G^{(j)}=G^{(j)} U=N G^{(j)}, j=1,2$;

(2) $x^{T}(U \otimes P) y=-\sum_{i=1}^{N-1} \sum_{j=i+1}^{N} u_{i j}\left(x_{i}-x_{j}\right)^{T} P\left(y_{i}-y_{j}\right)$;

where $G^{(1)}$ and $G^{(2)}$ are defined in model (1).

Lemma 5: [43] Let $X, Y, \Xi$ be any matrices with $\Xi$ satisfying $\Xi^{T} \Xi \leq I$. For any given scalar $\varepsilon>0$, the following inequality holds:

$$
X \Xi Y+(X \Xi Y)^{T} \leq \varepsilon^{-1} X X^{T}+\varepsilon Y^{T} Y
$$

\section{MAin Results}

Before starting the main results, we introduce two more notations to facilitate the readers. Let $C^{1,2}(\mathbb{R} \times$ $\mathbb{R}^{n N}, \mathbb{R}^{+}$) denote the family of all nonnegative function $V(t, x)$ on $\mathbb{R} \times \mathbb{R}^{n N}$ which are continuously twice differentiable in $x$ and once differentiable in $t$. For each $V \in C^{1,2}\left(\mathbb{R} \times \mathbb{R}^{n N}, \mathbb{R}^{+}\right)$, by Itô's differential formula [9], the stochastic derivative of $V(t, x(t))$ along (11) can be obtained as:

$$
\begin{aligned}
d V(t, x(t))= & \mathcal{L} V(t, x(t)) d t+V_{x}(t, x(t))\left[\sigma(t, x(t), x(t-\tau(t))) d \omega_{3}(t)\right. \\
& \left.+\left(G^{(1)} \otimes \Gamma_{1}\right) x(t) d \omega_{1}(t)+\left(G^{(2)} \otimes \Gamma_{2}\right) x(t-\tau(t)) d \omega_{2}(t)\right], \quad t>0
\end{aligned}
$$

where the weak infinitesimal operator $\mathcal{L}$ of the stochastic process $\left\{x_{t}=x(t+s) \mid t \geq 0,-\tau_{M} \leq s \leq 0\right\}$ is given by

$$
\begin{aligned}
\mathcal{L} V(t, x(t))= & V_{t}(t, x(t))+V_{x}(t, x(t))\left[\left(I_{N} \otimes \bar{A}+G^{(1)} \otimes \Gamma_{1}\right) x(t)+\left(I_{N} \otimes \bar{D}+G^{(2)} \otimes \Gamma_{2}\right) x(t-\tau(t))\right. \\
& \left.+\left(I_{N} \otimes \bar{B}\right) F(x(t))\right]+\frac{1}{2} \operatorname{trace}\left[\sigma^{T}(t, x(t), x(t-\tau(t))) V_{x x}(t, x(t)) \sigma(t, x(t), x(t-\tau(t)))\right. \\
& +x^{T}(t)\left(G^{(1)} \otimes \Gamma_{1}^{T}\right) V_{x x}(t, x(t))\left(G^{(1)} \otimes \Gamma_{1}\right) x(t) \\
& \left.+x^{T}(t-\tau(t))\left(G^{(2)} \otimes \Gamma_{2}^{T}\right) V_{x x}(t, x(t))\left(G^{(2)} \otimes \Gamma_{2}\right) x(t-\tau(t))\right] .
\end{aligned}
$$

Our main results are given in the following theorem.

Theorem 1: Under Assumption 1, the complex network (11) is globally robustly synchronized in the mean square, if there exist five matrices $P>0, Q_{1}>0, Q_{2}>0, Q_{3}>0, Q_{4}>0$, two matrices $T_{1}, T_{2}$ and four scalars $\lambda>0, \beta>0, \varepsilon_{1}>0, \varepsilon_{2}>0$ such that the following LMIs hold for all $1 \leq i<j \leq N$ :

$$
P<\lambda I
$$




$$
\left[\begin{array}{cccccccccc}
\Phi_{11}^{(i, j)} & \Phi_{12}^{(i, j)} & \Phi_{13} & \Phi_{14}^{(i, j)} & \Phi_{15}^{(i, j)} & \tau_{M} T_{1} B & \varepsilon_{1} M_{1}^{T} & 0 & P E & T_{1} E \\
* & \Phi_{22}^{(i, j)} & 0 & \Phi_{24}^{(i, j)} & \Phi_{25}^{(i, j)} & \tau_{M} T_{2} B & \varepsilon_{1} M_{2}^{T} & 0 & 0 & T_{2} E \\
* & * & \tau_{M} Q_{4}-2 \beta I & 0 & 0 & 0 & \varepsilon_{1} M_{3}^{T} & 0 & 0 & 0 \\
* & * & * & -\tau_{M} Q_{2} & 0 & 0 & 0 & \varepsilon_{2} M_{1}^{T} & 0 & 0 \\
* & * & * & * & -\tau_{M} Q_{3} & 0 & 0 & \varepsilon_{2} M_{2}^{T} & 0 & 0 \\
* & * & * & * & * & -\tau_{M} Q_{4} & 0 & \varepsilon_{2} M_{3}^{T} & 0 & 0 \\
* & * & * & * & * & * & -\varepsilon_{1} I & 0 & 0 & 0 \\
* & * & * & * & * & * & * & -\varepsilon_{2} I & 0 & 0 \\
* & * & * & * & * & * & * & * & -\varepsilon_{1} I & 0 \\
* & * & * & * & * & * & * & * & * & -\varepsilon_{2} I
\end{array}\right]<0,
$$

where

$$
\begin{aligned}
\Phi_{11}^{(i, j)}= & P A+A^{T} P-N G_{i j}^{(1)}\left(P \Gamma_{1}+\Gamma_{1}^{T} P\right)-N G_{i j}^{(1,1)} \Gamma_{1}^{T} P \Gamma_{1}+Q_{1}+\tau_{M} Q_{2}-T_{1}-T_{1}^{T} \\
& +\lambda W_{1}^{T} W_{1}-\beta\left(X_{1}^{T} X_{2}+X_{2}^{T} X_{1}\right), \\
\Phi_{22}^{(i, j)}= & -N G_{i j}^{(2,2)} \Gamma_{2}^{T} P \Gamma_{2}-(1-\mu) Q_{1}+\tau_{M} Q_{3}+T_{2}+T_{2}^{T}+\lambda W_{2}^{T} W_{2}, \\
\Phi_{12}^{(i, j)}= & P D-N G_{i j}^{(2)} P \Gamma_{2}+T_{1}-T_{2}^{T}, \Phi_{13}=P B+\beta\left(X_{1}^{T}+X_{2}^{T}\right), \Phi_{14}^{(i, j)}=\tau_{M} T_{1} A-N \tau_{M} G_{i j}^{(1)} T_{1} \Gamma_{1}, \\
\Phi_{15}^{(i, j)}= & \tau_{M} T_{1} D-N \tau_{M} G_{i j}^{(2)} T_{1} \Gamma_{2}, \Phi_{24}^{(i, j)}=\tau_{M} T_{2} A-N \tau_{M} G_{i j}^{(1)} T_{2} \Gamma_{1}, \Phi_{25}^{(i, j)}=\tau_{M} T_{2} D-N \tau_{M} G_{i j}^{(2)} T_{2} \Gamma_{2}
\end{aligned}
$$

and $G^{(1,1)}=G^{(1)} G^{(1)}=\left(G_{i j}^{(1,1)}\right)_{N \times N}, G^{(2,2)}=G^{(2)} G^{(2)}=\left(G_{i j}^{(2,2)}\right)_{N \times N}$.

Proof: Pre- and post-multiplying the inequality (15) by the block-diagonal matrix

$$
\operatorname{diag}\left(I, I, I, \frac{1}{\tau_{M}}, \frac{1}{\tau_{M}}, \frac{1}{\tau_{M}}, I, I, I, I\right)
$$

and by Lemma 2, the inequality is equivalent to

$$
\widehat{\Phi}_{i j}=\Phi_{i j}+\varepsilon_{2}^{-1} \hat{T}_{12} E E^{T} \hat{T}_{12}^{T}+\varepsilon_{1}^{-1} \hat{P} E E^{T} \hat{P}^{T}+\varepsilon_{1} \bar{M}_{1} \bar{M}_{1}^{T}+\varepsilon_{2} \hat{M}_{1} \hat{M}_{1}^{T}<0,
$$

where

$$
\Phi_{i j}=\left[\begin{array}{cccccc}
\Phi_{11}^{(i, j)} & \Phi_{12}^{(i, j)} & P B+\beta\left(X_{1}^{T}+X_{2}^{T}\right) & T_{1} A-N G_{i j}^{(1)} T_{1} \Gamma_{1} & T_{1} D-N G_{i j}^{2} T_{1} \Gamma_{2} & T_{1} B \\
* & \Phi_{22}^{(i, j)} & 0 & T_{2} A-N G_{i j}^{(1)} T_{2} \Gamma_{1} & T_{2} D-N G_{i j}^{(2)} T_{2} \Gamma_{2} & T_{2} B \\
* & * & \tau_{M} Q_{4}-2 \beta I & 0 & 0 & 0 \\
* & * & * & -\frac{1}{\tau_{M}} Q_{2} & 0 & 0 \\
* & * & * & * & -\frac{1}{\tau_{M}} Q_{3} & 0 \\
* & * & * & * & * & -\frac{1}{\tau_{M}} Q_{4}
\end{array}\right],
$$

and

$$
\hat{P}=\left[\begin{array}{c}
P \\
0 \\
0 \\
0 \\
0 \\
0
\end{array}\right], \quad \hat{T}_{12}=\left[\begin{array}{c}
T_{1} \\
T_{2} \\
0 \\
0 \\
0 \\
0
\end{array}\right], \quad \bar{M}_{1}=\left[\begin{array}{c}
M_{1}^{T} \\
M_{2}^{T} \\
M_{3}^{T} \\
0 \\
0 \\
0
\end{array}\right], \quad \hat{M}_{1}=\left[\begin{array}{c}
0 \\
0 \\
0 \\
M_{1}^{T} \\
M_{2}^{T} \\
M_{3}^{T}
\end{array}\right] .
$$

Define a Lyapunov functional candidate $V(t, x(t)) \in C^{1,2}\left(\mathbb{R} \times \mathbb{R}^{n N}, \mathbb{R}^{+}\right)$by

$$
V(t, x(t))=V_{1}(t, x(t))+V_{2}(t, x(t))+V_{3}(t, x(t))+V_{4}(t, x(t)), \quad t>0
$$


where

$$
\begin{aligned}
V_{1}(t, x(t)) & =x^{T}(t)(U \otimes P) x(t), \\
V_{2}(t, x(t)) & =\int_{t-\tau(t)}^{t} x^{T}(s)\left(U \otimes Q_{1}\right) x(s) d s+\int_{-\tau_{M}}^{0} \int_{\theta}^{0} x^{T}(t+s)\left(U \otimes Q_{2}\right) x(t+s) d s d \theta, \\
V_{3}(t, x(t)) & =\int_{t-\tau_{M}}^{t} \int_{\theta}^{t} x^{T}(s-\tau(s))\left(U \otimes Q_{3}\right) x(s-\tau(s)) d s d \theta, \\
V_{4}(t, x(t)) & =\int_{t-\tau_{M}}^{t} \int_{\theta}^{t} F^{T}(x(s))\left(U \otimes Q_{4}\right) F(x(s)) d s d \theta
\end{aligned}
$$

and the $N \times N$ matrix $U$ is defined in Lemma 4 .

By Itô's differential rule, the stochastic derivative of $V_{i}(i=1,2,3,4)$ along the trajectory of complex networks (11) can be obtained as follows:

$$
\begin{aligned}
d V_{1}(t, x(t))= & \left\{x^{T}(t)\left[U \otimes\left(P \bar{A}+\bar{A}^{T} P\right)+N G^{(1)} \otimes\left(P \Gamma_{1}+\Gamma_{1}^{T} P\right)+N G^{(1,1)} \otimes\left(\Gamma_{1}^{T} P \Gamma_{1}\right)\right] x(t)\right. \\
& 2 x^{T}(t)\left[U \otimes(P \bar{D})+N G^{(2)} \otimes\left(P \Gamma_{2}\right)\right] x(t-\tau(t))+2 x^{T}(t)(U \otimes(P \bar{B})) F(x(t)) \\
& +\sigma^{T}(t, x(t), x(t-\tau(t)))(U \otimes P) \sigma(t, x(t), x(t-\tau(t))) \\
& \left.+x^{T}(t-\tau(t))\left(N G^{(2,2)} \otimes\left(\Gamma_{2}^{T} P \Gamma_{2}\right)\right) x(t-\tau(t))\right\} d t \\
& +2 x^{T}(t)(U \otimes P)\left[\sigma(t, x(t), x(t-\tau(t))) d \omega_{3}(t)\right. \\
& \left.+\left(G^{(1)} \otimes \Gamma_{1}\right) x(t) d \omega_{1}(t)+\left(G^{(2)} \otimes \Gamma_{2}\right) x(t-\tau(t)) d \omega_{2}(t)\right]
\end{aligned}
$$

here, in the second step of (22), we have used the properties of Kronecker product in Lemma 1.

$$
\begin{aligned}
d V_{2}(t, x(t))= & {\left[x^{T}(t)\left(U \otimes Q_{1}\right) x(t)-(1-\dot{\tau}(t)) x^{T}(t-\tau(t))\left(U \otimes Q_{1}\right) x(t-\tau(t))\right.} \\
& \left.+\tau_{M} x^{T}(t)\left(U \otimes Q_{2}\right) x(t)-\int_{t-\tau_{M}}^{t} x^{T}(s)\left(U \otimes Q_{2}\right) x(s) d s\right] d t \\
\leq & {\left[x^{T}(t)\left(U \otimes\left(Q_{1}+\tau_{M} Q_{2}\right)\right) x(t)-(1-\mu) x^{T}(t-\tau(t))\left(U \otimes Q_{1}\right) x(t-\tau(t))\right.} \\
& \left.-\frac{1}{\tau_{M}}\left(\int_{t-\tau(t)}^{t} x(s) d s\right)^{T}\left(U \otimes Q_{2}\right)\left(\int_{t-\tau(t)}^{t} x(s) d s\right)\right] d t .
\end{aligned}
$$

Note that, in obtaining (23), we have utilized the condition $\dot{\tau}(t) \leq \mu, \tau(t) \leq \tau_{M}$ and the Jensen inequality (12) from Lemma 3 in the second step of (23). Similarly, by Lemma 3, one has

$$
\begin{aligned}
d V_{3}(t, x(t)) \leq & {\left[\tau_{M} x^{T}(t-\tau(t))\left(U \otimes Q_{3}\right) x(t-\tau(t))\right.} \\
& \left.-\frac{1}{\tau_{M}}\left(\int_{t-\tau(t)}^{t} x(s-\tau(s)) d s\right)^{T}\left(U \otimes Q_{3}\right)\left(\int_{t-\tau(t)}^{t} x(s-\tau(s)) d s\right)\right] d t ; \\
d V_{4}(t, x(t)) \leq & {\left[\tau_{M} F^{T}(x(t))\left(U \otimes Q_{4}\right) F(x(t))\right.} \\
& \left.-\frac{1}{\tau_{M}}\left(\int_{t-\tau(t)}^{t} F(x(s)) d s\right)^{T}\left(U \otimes Q_{4}\right)\left(\int_{t-\tau(t)}^{t} F(x(s)) d s\right)\right] d t .
\end{aligned}
$$

On the other hand, it follows that for any matrices $T_{1}$ and $T_{2}$,

$$
2\left[x^{T}(t)\left(U \otimes T_{1}\right)+x^{T}(t-\tau(t))\left(U \otimes T_{2}\right)\right]\left(-x(t)+x(t-\tau(t))+\int_{t-\tau(t)}^{t} d x(s)\right)=0, \quad \forall t>0 .
$$


Substituting (11) into (26) gives

$$
\begin{aligned}
0= & -x^{T}(t)\left(U \otimes\left(T_{1}+T_{1}^{T}\right)\right) x(t)+x^{T}(t-\tau(t))\left(U \otimes\left(T_{2}+T_{2}^{T}\right)\right) x(t-\tau(t)) \\
& +2 x^{T}(t)\left(U \otimes\left(T_{1}-T_{2}^{T}\right)\right) x(t-\tau(t))+2 x^{T}(t)\left[U \otimes\left(T_{1} \bar{A}\right)+N G^{(1)} \otimes\left(T_{1} \Gamma_{1}\right)\right] \int_{t-\tau(t)}^{t} x(s) d s \\
& +2 x^{T}(t-\tau(t))\left[U \otimes\left(T_{2} \bar{A}\right)+N G^{(1)} \otimes\left(T_{2} \Gamma_{1}\right)\right] \int_{t-\tau(t)}^{t} x(s) d s \\
& +2 x^{T}(t)\left[U \otimes\left(T_{1} \bar{D}\right)+N G^{(2)} \otimes\left(T_{1} \Gamma_{2}\right)\right] \int_{t-\tau(t)}^{t} x(s-\tau(s)) d s \\
& +2 x^{T}(t-\tau(t))\left[U \otimes\left(T_{2} \bar{D}\right)+N G^{(2)} \otimes\left(T_{2} \Gamma_{2}\right)\right] \int_{t-\tau(t)}^{t} x(s-\tau(s)) d s \\
& +2 x^{T}(t)\left(U \otimes\left(T_{1} \bar{B}\right)\right) \int_{t-\tau(t)}^{t} F(x(s)) d s+2 x^{T}(t-\tau(t))\left(U \otimes\left(T_{2} \bar{B}\right)\right) \int_{t-\tau(t)}^{t} F(x(s)) d s \\
& +2\left[x^{T}(t)\left(U \otimes T_{1}\right)+x^{T}(t-\tau(t))\left(U \otimes T_{2}\right)\right] \int_{t-\tau(t)}^{t}\left[\sigma(s, x(s), x(s-\tau(s))) d \omega_{3}(s)\right. \\
& \left.+\left(G^{(1)} \otimes \Gamma_{1}\right) x(s) d \omega_{1}(s)+\left(G^{(2)} \otimes \Gamma_{2}\right) x(s-\tau(s)) d \omega_{2}(s)\right], \quad t>0 .
\end{aligned}
$$

Therefore, combining (22)-(25) together with (27) and by Lemma 4, we have

$$
\begin{aligned}
d V(t, x(t)) \leq & \sum_{i=1}^{N-1} \sum_{j=i+1}^{N}\left\{( x _ { i } ( t ) - x _ { j } ( t ) ) ^ { T } \left[P \bar{A}+\bar{A}^{T} P-N G_{i j}^{(1)}\left(P \Gamma_{1}+\Gamma_{1}^{T} P\right)-N G_{i j}^{(1,1)} \Gamma_{1}^{T} P \Gamma_{1}\right.\right. \\
& \left.+Q_{1}+\tau_{M} Q_{2}-T_{1}-T_{1}^{T}\right]\left(x_{i}(t)-x_{j}(t)\right)+\left(x_{i}(t-\tau(t))-x_{j}(t-\tau(t))\right)^{T}\left[-N G_{i j}^{(2,2)} \Gamma_{2}^{T} P \Gamma_{2}\right. \\
& \left.-(1-\mu) Q_{1}+\tau_{M} Q_{3}+T_{2}+T_{2}^{T}\right]\left(x_{i}(t-\tau(t))-x_{j}(t-\tau(t))\right) \\
& +\left(f\left(x_{i}(t)\right)-f\left(x_{j}(t)\right)\right)^{T}\left(\tau_{M} Q_{4}\right)\left(f\left(x_{i}(t)\right)-f\left(x_{j}(t)\right)\right) \\
& +\left(\int_{t-\tau(t)}^{t}\left(x_{i}(s)-x_{j}(s)\right) d s\right)^{T}\left(-\frac{1}{\tau_{M}} Q_{2}\right)\left(\int_{t-\tau(t)}^{t}\left(x_{i}(s)-x_{j}(s)\right) d s\right) \\
& +\left(\int_{t-\tau(t)}^{t}\left(x_{i}(s-\tau(s))-x_{j}(s-\tau(s))\right) d s\right)^{T}\left(-\frac{1}{\tau_{M}} Q_{3}\right)\left(\int_{t-\tau(t)}^{t}\left(x_{i}(s-\tau(s))-x_{j}(s-\tau(s))\right) d s\right) \\
& +\left(\int_{t-\tau(t)}^{t}\left(f\left(x_{i}(s)\right)-f\left(x_{j}(s)\right)\right) d s\right)^{T}\left(-\frac{1}{\tau_{M}} Q_{4}\right)\left(\int_{t-\tau(t)}^{t}\left(f\left(x_{i}(s)\right)-f\left(x_{j}(s)\right)\right) d s\right) \\
& +2\left(x_{i}(t)-x_{j}(t)\right)^{T}\left[\left(P \bar{D}-N G_{i j}^{(2)} P \Gamma_{2}+T_{1}-T_{2}^{T}\right)\left(x_{i}(t-\tau(t))-x_{j}(t-\tau(t))\right)\right. \\
& +P \bar{B}\left(f\left(x_{i}(t)\right)-f\left(x_{j}(t)\right)\right)+\left(T_{1} \bar{D}-N G_{i j}^{(2)} T_{1} \Gamma_{2}\right) \int_{t-\tau(t)}^{t}\left(x_{i}(s-\tau(s))-x_{j}(s-\tau(s))\right) d s \\
& \left.+\left(T_{1} \bar{A}-N G_{i j}^{(1)} T_{1} \Gamma_{1}\right) \int_{t-\tau(t)}^{t}\left(x_{i}(s)-x_{j}(s)\right) d s+T_{1} \bar{B} \int_{t-\tau(t)}^{t}\left(f\left(x_{i}(s)\right)-f\left(x_{j}(s)\right)\right) d s\right] \\
& +2\left(x_{i}(t-\tau(t))-x_{j}(t-\tau(t))\right)^{T}\left[\left(T_{2} \bar{D}-N G_{i j}^{(2)} T_{2} \Gamma_{2}\right) \int_{t-\tau(t)}^{t}\left(x_{i}(s-\tau(s))-x_{j}(s-\tau(s))\right) d s\right. \\
& \left.+\left(T_{2} \bar{A}-N G_{i j}^{(1)} T_{2} \Gamma_{1}\right) \int_{t-\tau(t)}^{t}\left(x_{i}(s)-x_{j}(s)\right) d s+T_{2} \bar{B} \int_{t-\tau(t)}^{t}\left(f\left(x_{i}(s)\right)-f\left(x_{j}(s)\right)\right) d s\right] \\
& +\left[\sigma_{i}(t, x(t), x(t-\tau(t)))-\sigma_{j}(t, x(t), x(t-\tau(t)))\right]^{T} P \\
& \left.\times\left[\sigma_{i}(t, x(t), x(t-\tau(t)))-\sigma_{j}(t, x(t), x(t-\tau(t)))\right]\right\} d t \\
& \\
& \\
&
\end{aligned}
$$




$$
\begin{aligned}
& +2 x^{T}(t)\left[(U \otimes P) \sigma(t, x(t), x(t-\tau(t))) d \omega_{3}(t)+\left(N G^{(2)} \otimes\left(P \Gamma_{2}\right)\right) x(t-\tau(t)) d \omega_{2}(t)\right. \\
& \left.+\left(N G^{(1)} \otimes\left(P \Gamma_{1}\right)\right) x(t) d \omega_{1}(t)\right]+2\left[x^{T}(t)\left(U \otimes T_{1}\right)+x^{T}(t-\tau(t))\left(U \otimes T_{2}\right)\right] \\
& \times \int_{t-\tau(t)}^{t}\left[\sigma(s, x(s), x(s-\tau(s))) d \omega_{3}(s)\right. \\
& \left.+\left(G^{(1)} \otimes \Gamma_{1}\right) x(s) d \omega_{1}(s)+\left(G^{(2)} \otimes \Gamma_{2}\right) x(s-\tau(s)) d \omega_{2}(s)\right]
\end{aligned}
$$

Conditions (6) and (14) ensure that

$$
\begin{aligned}
& {\left[\sigma_{i}(t, x(t), x(t-\tau(t)))-\sigma_{j}(t, x(t), x(t-\tau(t)))\right]^{T} P\left[\sigma_{i}(t, x(t), x(t-\tau(t)))-\sigma_{j}(t, x(t), x(t-\tau(t)))\right] } \\
\leq \quad & \lambda\left[\left(x_{i}(t)-x_{j}(t)\right)^{T} W_{1}^{T} W_{1}\left(x_{i}(t)-x_{j}(t)\right)\right. \\
& \left.+\left(x_{i}(t-\tau(t))-x_{j}(t-\tau(t))\right)^{T} W_{2}^{T} W_{2}\left(x_{i}(t-\tau(t))-x_{j}(t-\tau(t))\right)\right], \quad 1 \leq i<j \leq N, t \in \mathbb{R}^{+} .
\end{aligned}
$$

At the same time, it follows from Assumption 1 that for all $t \in \mathbb{R}^{+}$and $1 \leq i<j \leq N$ :

$$
\beta\left[\begin{array}{c}
x_{i}(t)-x_{j}(t) \\
f\left(x_{i}(t)\right)-f\left(x_{j}(t)\right)
\end{array}\right]^{T}\left[\begin{array}{cc}
X_{1}^{T} X_{2}+X_{2}^{T} X_{1} & -\left(X_{1}^{T}+X_{2}^{T}\right) \\
* & 2 I
\end{array}\right]\left[\begin{array}{c}
x_{i}(t)-x_{j}(t) \\
f\left(x_{i}(t)\right)-f\left(x_{j}(t)\right)
\end{array}\right] \leq 0 .
$$

Using (28)-(30), we get

$$
\begin{aligned}
d V(t, x(t)) \leq & \sum_{i=1}^{N-1} \sum_{j=i+1}^{N} \xi_{i j}^{T}(t)\left(\Phi_{i j}+\Delta \Phi\right) \xi_{i j}(t)+2 x^{T}(t)\left[(U \otimes P) \sigma(t, x(t), x(t-\tau(t))) d \omega_{3}(t)\right. \\
& \left.+\left(N G^{(2)} \otimes\left(P \Gamma_{2}\right)\right) x(t-\tau(t)) d \omega_{2}(t)+\left(N G^{(1)} \otimes\left(P \Gamma_{1}\right)\right) x(t) d \omega_{1}(t)\right] \\
& +2\left[x^{T}(t)\left(U \otimes T_{1}\right)+x^{T}(t-\tau(t))\left(U \otimes T_{2}\right)\right] \times \int_{t-\tau(t)}^{t}\left[\sigma(s, x(s), x(s-\tau(s))) d \omega_{3}(s)\right. \\
& \left.+\left(G^{(1)} \otimes \Gamma_{1}\right) x(s) d \omega_{1}(s)+\left(G^{(2)} \otimes \Gamma_{2}\right) x(s-\tau(s)) d \omega_{2}(s)\right]
\end{aligned}
$$

where $\xi_{i j}(t)=\left[\left(x_{i}(t)-x_{j}(t)\right)^{T},\left(x_{i}(t-\tau(t))-x_{j}(t-\tau(t))\right)^{T},\left(f\left(x_{i}(t)\right)-f\left(x_{j}(t)\right)\right)^{T},\left(\int_{t-\tau(t)}^{t}\left(x_{i}(s)-x_{j}(s)\right) d s\right)^{T}\right.$, $\left.\left(\int_{t-\tau(t)}^{t}\left(x_{i}(s-\tau(s))-x_{j}(s-\tau(s))\right) d s\right)^{T},\left(\int_{t-\tau(t)}^{t}\left(f\left(x_{i}(s)\right)-f\left(x_{j}(s)\right)\right) d s\right)^{T}\right]^{T}$,

$$
\begin{aligned}
\Delta \Phi & =\left[\begin{array}{cccccc}
P \Delta A+(\Delta A)^{T} P & P \Delta D & P \Delta B & T_{1} \Delta A & T_{1} \Delta D & T_{1} \Delta B \\
* & 0 & 0 & T_{2} \Delta A & T_{2} \Delta D & T_{2} \Delta B \\
* & * & 0 & 0 & 0 & 0 \\
* & * & * & 0 & 0 & 0 \\
* & * & * & * & 0 & 0 \\
* & * & * & * & * & 0
\end{array}\right] \\
& =\hat{P} \aleph_{1}^{T}+\aleph_{1} \hat{P}^{T}+\hat{T}_{12} \aleph_{2}^{T}+\aleph_{2} \hat{T}_{12}^{T}
\end{aligned}
$$

and $\aleph_{1}^{T}=\left[\begin{array}{llllll}\Delta A & \Delta D & \Delta B & 0 & 0 & 0\end{array}\right], \aleph_{2}^{T}=\left[\begin{array}{llllll}0 & 0 & 0 & \Delta A & \Delta D & \Delta B\end{array}\right]$.

From (2), one knows that

$$
\aleph_{1}^{T}=E \mathcal{F} \bar{M}_{1}^{T}, \quad \aleph_{2}^{T}=E \mathcal{F} \hat{M}_{1}^{T} ;
$$

and therefore it follows from Lemma 5 that

$$
\Delta \Phi \leq \varepsilon_{1}^{-1} \hat{P} E E^{T} \hat{P}^{T}+\varepsilon_{1} \bar{M}_{1} \bar{M}_{1}^{T}+\varepsilon_{2}^{-1} \hat{T}_{12} E E^{T} \hat{T}_{12}^{T}+\varepsilon_{2} \hat{M}_{1} \hat{M}_{1}^{T} .
$$


Taking the mathematical expectation of both sides of (31) and considering (5), it can be derived that

$$
\begin{aligned}
\frac{\mathbb{E}\{d V(t, x(t))\}}{d t} & \leq \sum_{i=1}^{N-1} \sum_{j=i+1}^{N} \mathbb{E}\left\{\xi_{i j}^{T}(t)\left(\Phi_{i j}+\Delta \Phi\right) \xi_{i j}(t)\right\} \\
& \leq \sum_{i=1}^{N-1} \sum_{j=i+1}^{N} \mathbb{E}\left\{\xi_{i j}^{T}(t) \widehat{\Phi} \xi_{i j}(t)\right\} \leq-\alpha \mathbb{E}\left\{\left\|x_{i}(t)-x_{j}(t)\right\|^{2}\right\}, \quad t>0
\end{aligned}
$$

for some constant $\alpha>0$. This indicates from the Lyapunov stability theory [30] that the dynamics of the complex networks (11) is globally robustly synchronized in the mean square. This completes the proof of Theorem 1.

Remark 3: In Theorem 1, a delay-dependent criterion is established that ensures that the coupled complex network is globally robustly synchronized in the mean square for all admissible parameter uncertainties, and the criterion is expressed in terms of the solution to certain LMIs. Note that LMIs can be effectively solved and checked by the algorithms such as the interior-point method [1].

Remark 4: In [4], the global synchronization problem has been studied for an array of linearly stochastically coupled networks with time delays, where the subsystems are coupled stochastically. In this paper, we have taken two significant steps further. First, the stochastic disturbances are considered not only in the coupling terms (i.e., the coupled terms are subjected to stochastic disturbances) but also in the subsystems. Second, the parameter uncertainties are taken into account. Accordingly, our main result is more general than that of $[4]$.

In the following, we show that the main criterion can be easily specified to some special cases.

Case 1. Let's assume that there are no parameter uncertainties in $A, D$ and $B$, that is, the coupled network system is simplified to

$$
\begin{aligned}
d x_{i}(t)= & {\left[A x_{i}(t)+D x_{i}(t-\tau(t))+B f\left(x_{i}(t)\right)\right] d t+\sigma_{i}\left(t, x_{i}(t), x_{i}(t-\tau(t))\right) d \omega_{3}(t) } \\
& +\sum_{j=1}^{N} G_{i j}^{(1)} \Gamma_{1} x_{j}(t)\left(d t+d \omega_{1}(t)\right)+\sum_{j=1}^{N} G_{i j}^{(2)} \Gamma_{2} x_{j}(t-\tau(t))\left(d t+d \omega_{2}(t)\right), \quad t>0 .
\end{aligned}
$$

Then we have the following corollary.

Corollary 1: Under Assumption 1, the complex network (34) is globally synchronized in the mean square, if there exist five matrices $P>0, Q_{1}>0, Q_{2}>0, Q_{3}>0, Q_{4}>0$, two matrices $T_{1}, T_{2}$ and two scalars $\lambda>0$, $\beta>0$ such that the following LMIs hold for all $1 \leq i<j \leq N$ :

$$
\begin{gathered}
P<\lambda I, \\
{\left[\begin{array}{cccccc}
\Phi_{11}^{(i, j)} & \Phi_{12}^{(i, j)} & P B+\beta\left(X_{1}^{T}+X_{2}^{T}\right) & \tau_{M}\left(T_{1} A-N G_{i j}^{(1)} T_{1} \Gamma_{1}\right) & \tau_{M}\left(T_{1} D-N G_{i j}^{(2)} T_{1} \Gamma_{2}\right) & \tau_{M} T_{1} B \\
* & \Phi_{22}^{(i, j)} & 0 & \tau_{M}\left(T_{2} A-N G_{i j}^{(1)} T_{2} \Gamma_{1}\right) & \tau_{M}\left(T_{2} D-N G_{i j}^{(2)} T_{2} \Gamma_{2}\right) & \tau_{M} T_{2} B \\
* & * & \tau_{M} Q_{4}-2 \beta I & 0 & 0 & 0 \\
* & * & * & -\tau_{M} Q_{2} & 0 & 0 \\
* & * & * & * & -\tau_{M} Q_{3} & 0 \\
* & * & * & * & * & -\tau_{M} Q_{4}
\end{array}\right]<0}
\end{gathered}
$$

where $\Phi_{11}^{(i, j)}, \Phi_{22}^{(i, j)}$ and $\Phi_{12}^{(i, j)}$ are defined as in Theorem 1 .

Case 2. Assume now that there are no stochastic disturbances in (1) and the network model reduces to

$$
\begin{aligned}
d x_{i}(t)= & {\left[(A+\Delta A) x_{i}(t)+(D+\Delta D) x_{i}(t-\tau(t))+(B+\Delta B) f\left(x_{i}(t)\right)\right.} \\
& \left.+\sum_{j=1}^{N} G_{i j}^{(1)} \Gamma_{1} x_{j}(t)+\sum_{j=1}^{N} G_{i j}^{(2)} \Gamma_{2} x_{j}(t-\tau(t))\right] d t, \quad t>0 .
\end{aligned}
$$


Then the following result is true.

Corollary 2: Under Assumption 1, the complex network (37) is globally robustly synchronized, if there exist five matrices $P>0, Q_{1}>0, Q_{2}>0, Q_{3}>0, Q_{4}>0$, two matrices $T_{1}, T_{2}$ and three scalars $\beta>0, \varepsilon_{1}>0$, $\varepsilon_{2}>0$ such that the following LMIs hold for all $1 \leq i<j \leq N$ :

$$
\left[\begin{array}{cccccccccc}
\Psi_{11}^{(i, j)} & \Phi_{12}^{(i, j)} & \Phi_{13} & \Phi_{14}^{(i, j)} & \Phi_{15}^{(i, j)} & \tau_{M} T_{1} B & \varepsilon_{1} M_{1}^{T} & 0 & P E & T_{1} E \\
* & \Psi_{22}^{(i, j)} & 0 & \Phi_{24}^{(i, j)} & \Phi_{25}^{(i, j)} & \tau_{M} T_{2} B & \varepsilon_{1} M_{2}^{T} & 0 & 0 & T_{2} E \\
* & * & \tau_{M} Q_{4}-2 \beta I & 0 & 0 & 0 & \varepsilon_{1} M_{3}^{T} & 0 & 0 & 0 \\
* & * & * & -\tau_{M} Q_{2} & 0 & 0 & 0 & \varepsilon_{2} M_{1}^{T} & 0 & 0 \\
* & * & * & * & -\tau_{M} Q_{3} & 0 & 0 & \varepsilon_{2} M_{2}^{T} & 0 & 0 \\
* & * & * & * & * & -\tau_{M} Q_{4} & 0 & \varepsilon_{2} M_{3}^{T} & 0 & 0 \\
* & * & * & * & * & * & -\varepsilon_{1} I & 0 & 0 & 0 \\
* & * & * & * & * & * & * & -\varepsilon_{2} I & 0 & 0 \\
* & * & * & * & * & * & * & * & -\varepsilon_{1} I & 0 \\
* & * & * & * & * & * & * & * & * & -\varepsilon_{2} I
\end{array}\right]<0,
$$

where $\Psi_{11}^{(i, j)}=P A+A^{T} P-N G_{i j}^{(1)}\left(P \Gamma_{1}+\Gamma_{1}^{T} P\right)+Q_{1}+\tau_{M} Q_{2}-T_{1}-T_{1}^{T}-\beta\left(X_{1}^{T} X_{2}+X_{2}^{T} X_{1}\right), \Psi_{22}^{(i, j)}=$ $-(1-\mu) Q_{1}+\tau_{M} Q_{3}+T_{2}+T_{2}^{T}$ and the other symbols are as defined in Theorem 1.

Case 3. This time we assume that there are neither parameter uncertainties in $A, D$ and $B$ nor fixed coupling terms in (1), then the complex system is specialized to

$$
\begin{aligned}
d x_{i}(t)= & {\left[A x_{i}(t)+D x_{i}(t-\tau(t))+B f\left(x_{i}(t)\right)\right] d t+\sigma_{i}\left(t, x_{i}(t), x_{i}(t-\tau(t))\right) d \omega_{3}(t) } \\
& +\sum_{j=1}^{N} G_{i j}^{(1)} \Gamma_{1} x_{j}(t) d \omega_{1}(t)+\sum_{j=1}^{N} G_{i j}^{(2)} \Gamma_{2} x_{j}(t-\tau(t)) d \omega_{2}(t), \quad t>0
\end{aligned}
$$

and we can obtain the following corollary easily.

Corollary 3: Under Assumption 1, the complex network (39) is globally synchronized in the mean square, if there exist five matrices $P>0, Q_{1}>0, Q_{2}>0, Q_{3}>0, Q_{4}>0$, two matrices $T_{1}, T_{2}$ and two scalars $\lambda>0$, $\beta>0$ such that the following LMIs hold for all $1 \leq i<j \leq N$ :

$$
\left[\begin{array}{cccccc}
\Xi_{11}^{(i, j)} & P D+T_{1}-T_{2}^{T} & P B+\beta\left(X_{1}^{T}+X_{2}^{T}\right) & \tau_{M} T_{1} A & \tau_{M} T_{1} D & \tau_{M} T_{1} B \\
* & \Xi_{22}^{(i, j)} & 0 & \tau_{M} T_{2} A & \tau_{M} T_{2} D & \tau_{M} T_{2} B \\
* & * & \tau_{M} Q_{4}-2 \beta I & 0 & 0 & 0 \\
* & * & * & -\tau_{M} Q_{2} & 0 & 0 \\
* & * & * & * & -\tau_{M} Q_{3} & 0 \\
* & * & * & * & * & -\tau_{M} Q_{4}
\end{array}\right]<0,
$$

where $\Xi_{11}^{(i, j)}=P A+A^{T} P-N G_{i j}^{(1,1)} \Gamma_{1}^{T} P \Gamma_{1}+Q_{1}+\tau_{M} Q_{2}-T_{1}-T_{1}^{T}+\lambda W_{1}^{T} W_{1}-\beta\left(X_{1}^{T} X_{2}+X_{2}^{T} X_{1}\right)$ and $\Xi_{22}^{(i, j)}=-N G_{i j}^{(2,2)} \Gamma_{2}^{T} P \Gamma_{2}-(1-\mu) Q_{1}+\tau_{M} Q_{3}+T_{2}+T_{2}^{T}+\lambda W_{2}^{T} W_{2}$.

\section{NumericAl ExAmples}

In this section, two examples are illustrated to show the effectiveness of our results.

Example 1: Consider the following two-dimensional uncertain network with stochastic disturbances and time-varying delay:

$$
\begin{aligned}
d y(t)= & {[(A+\Delta A) y(t)+(D+\Delta D) y(t-\tau(t))+(B+\Delta B) f(y(t))] d t } \\
& +\sigma(t, y(t), y(t-\tau(t))) d \omega_{3}(t), \quad t>0
\end{aligned}
$$


where $y(t)=\left(y_{1}(t), y_{2}(t)\right)^{T} \in \mathbb{R}^{2}$ is the state vector of the network; the time-varying delay $\tau(t)=0.1+$ $0.05 \sin (20 t)$, that is, $\tau_{M}=0.15$ and $\mu=1$; the other parameters are as follows:

$$
A=\left[\begin{array}{cc}
-1.2 & 0.4 \\
0.4 & -1.6
\end{array}\right], \quad D=\left[\begin{array}{cc}
-0.4 & 1.0 \\
-1.4 & -0.2
\end{array}\right], \quad B=\left[\begin{array}{cc}
-0.6 & -0.1 \\
-0.3 & -1.5
\end{array}\right] .
$$

The parameter uncertainties $\Delta A, \Delta D$ and $\Delta B$ satisfy the conditions (2)-(3) with

$$
E=\left[\begin{array}{ll}
0.1 & 0.5 \\
0.5 & 0.3
\end{array}\right], \quad M_{1}=\left[\begin{array}{ll}
0.1 & -0.2 \\
0.1 & -0.1
\end{array}\right], \quad M_{2}=\left[\begin{array}{cc}
-0.2 & 0 \\
-0.3 & 0.2
\end{array}\right], \quad M_{3}=\left[\begin{array}{cc}
0.1 & 0.2 \\
-0.2 & -0.3
\end{array}\right]
$$

The nonlinear function $f(y(t))=\left(f_{1}\left(y_{1}(t)\right), f_{2}\left(y_{2}(t)\right)\right)^{T}$ with $f_{i}\left(y_{i}\right)=\tanh \left(y_{i}\right)(i=1,2)$; the noise intensity function vector $\sigma(\cdot, \cdot, \cdot)$ is of the following form:

$$
\sigma(t, y(t), y(t-\tau(t)))=\left[\begin{array}{cccc}
-0.1 & 0.1 & 0.2 & -0.2 \\
0.1 & -0.1 & 0.2 & -0.2
\end{array}\right]\left[\begin{array}{c}
y(t) \\
y(t-\tau(t))
\end{array}\right], \quad t \in \mathbb{R}^{+} .
$$

Obviously, the nonlinear functions satisfy Assumption 1 and condition (6) with

$$
X_{1}=\left[\begin{array}{ll}
0 & 0 \\
0 & 0
\end{array}\right], \quad X_{2}=\left[\begin{array}{ll}
1 & 0 \\
0 & 1
\end{array}\right] ; \quad W_{1}=\left[\begin{array}{cc}
-0.1 & 0.1 \\
0.1 & -0.1
\end{array}\right], \quad W_{2}=\left[\begin{array}{cc}
0.2 & -0.2 \\
0.2 & -0.2
\end{array}\right] .
$$

The dynamical behaviors of (42) with initial conditions as follows are shown in Figs. 1-2:

$$
y_{1}(s)=0.4, \quad y_{2}(s)=-0.2 ; \quad s \in[-0.15,0]
$$

where the uncertain matrix $\mathcal{F}$ is taken as a random variable satisfying the condition (3).

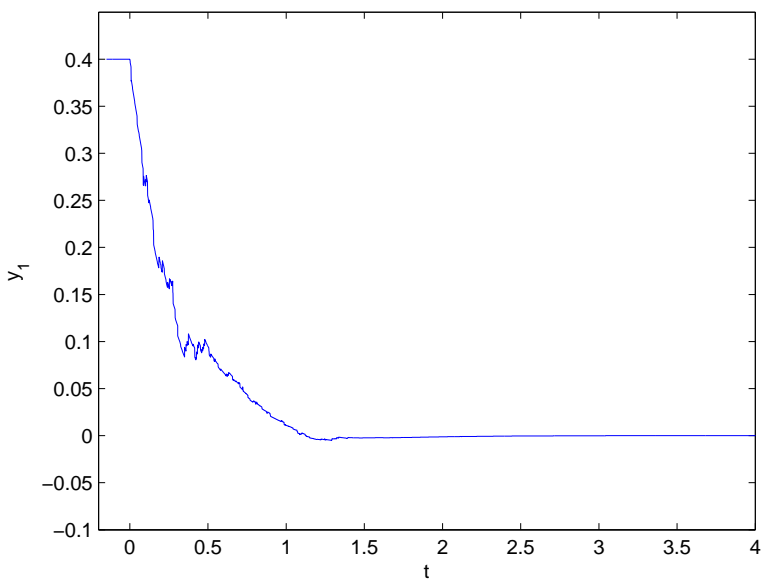

Fig. 1. State trajectory of $y_{1}(t)$ in model (42).

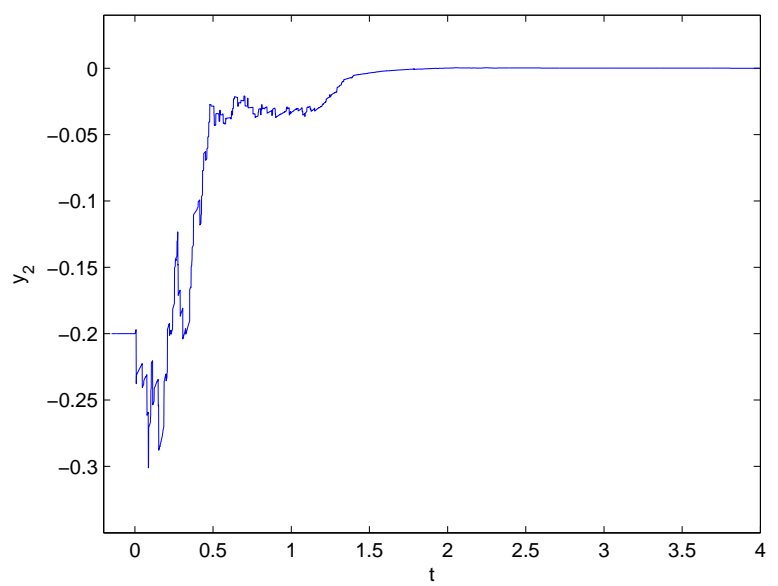

Fig. 2. State trajectory of $y_{2}(t)$ in model (42).

Now consider a coupled complex network consisting of three identical models (42). The state equations of the entire array are

$$
\begin{aligned}
d x_{i}(t)= & {\left[(A+\triangle A) x_{i}(t)+(D+\triangle D) x_{i}(t-\tau(t))+(B+\triangle B) f\left(x_{i}(t)\right)\right] d t } \\
& +\sigma\left(t, x_{i}(t), x_{i}(t-\tau(t))\right) d \omega_{3}(t) \\
& +\sum_{j=1}^{3} G_{i j}^{(1)} \Gamma_{1} x_{j}(t)\left(d t+d \omega_{1}(t)\right)+\sum_{j=1}^{3} G_{i j}^{(2)} \Gamma_{2} x_{j}(t-\tau(t))\left(d t+d \omega_{2}(t)\right)
\end{aligned}
$$


where $x_{i}(t)=\left(x_{i 1}(t), x_{i 2}(t)\right)^{T}(i=1,2,3)$ is the state vector of the $i$ th subsystem. Choose the coupling matrices $G^{(1)}, G^{(2)}$ and the linking matrices $\Gamma_{1}, \Gamma_{2}$ as

$G^{(1)}=\left[\begin{array}{ccc}-2 & 1 & 1 \\ 1 & -2 & 1 \\ 1 & 1 & -2\end{array}\right], G^{(2)}=\left[\begin{array}{ccc}-3 & 1 & 2 \\ 1 & -2 & 1 \\ 2 & 1 & -3\end{array}\right] ; \Gamma_{1}=\left[\begin{array}{cc}0.01 & 0.02 \\ -0.03 & 0.01\end{array}\right], \Gamma_{2}=\left[\begin{array}{cc}-0.02 & 0.05 \\ 0.07 & -0.05\end{array}\right]$.

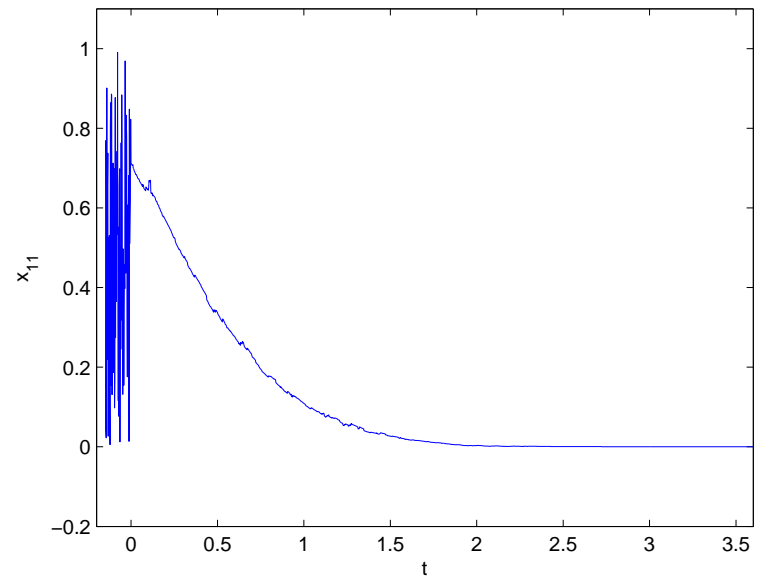

Fig. 3. State trajectory of $x_{11}(t)$ in model (44).

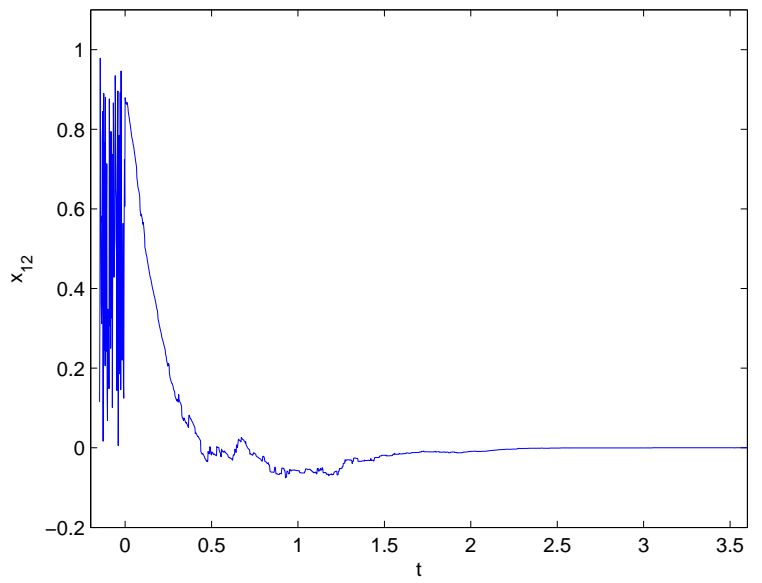

Fig. 4. State trajectory of $x_{12}(t)$ in model (44).

By using the Matlab LMI Toolbox, LMIs (14)-(15) can be solved with the feasible solutions as follows:

$$
\begin{gathered}
P=\left[\begin{array}{cc}
50.9053 & -6.8528 \\
-6.8528 & 27.7843
\end{array}\right], \quad Q_{1}=\left[\begin{array}{cc}
7.4118 & -0.4184 \\
-0.4184 & 3.7582
\end{array}\right], \quad Q_{2}=\left[\begin{array}{cc}
157.4762 & -66.7004 \\
-66.7004 & 129.3346
\end{array}\right], \\
Q_{3}=\left[\begin{array}{cc}
133.0133 & -19.4280 \\
-19.4280 & 59.9464
\end{array}\right], \quad Q_{4}=\left[\begin{array}{cc}
164.1311 & 24.4229 \\
24.4229 & 214.5054
\end{array}\right], \\
T_{1}=\left[\begin{array}{cc}
-17.1164 & -2.0297 \\
34.5245 & -9.6288
\end{array}\right], \quad T_{2}=\left[\begin{array}{cc}
-33.1419 & -4.8834 \\
26.6574 & -24.8379
\end{array}\right] ; \\
\varepsilon_{1}=48.9679, \quad \varepsilon_{2}=48.7753, \quad \beta=43.7551, \lambda=63.1424 .
\end{gathered}
$$

According to Theorem 1, the array of coupled uncertain networks (44) with stochastic disturbances can achieve globally robust synchronization in the mean square. The trajectories of system (44) are shown in Figs. 3-4 (for space consideration, only two of them are illustrated here)and the synchronization performances are illustrated in Figs. 5-6, where the initial states for (44) are taken randomly constants in $[0,1] \times[0,1]$. Figs. 3-6 conform that the dynamical system (44) is globally robustly synchronized in the mean square.

Example 2: The repressilator is a cyclic negative-feedback loop comprising three repressor genes (lacl, tetR, and $(l)$ and their promoters. The dynamics of the repressilator has been theoretically and experimentally investigated in EScherichia coli $[8,19]$. Specifically speaking, the kinetics of the system can be described as follows:

$$
\left\{\begin{aligned}
\dot{m}_{i} & =-m_{i}+\frac{\alpha}{1+p_{j}^{n}} \\
\dot{p}_{i} & =-\gamma\left(p_{i}-m_{i}\right)
\end{aligned}\right.
$$

where $i=l a c l$, tetR, $c l ; j=c l$, lacl, tetR. $m_{i}$ and $p_{i}$ are the concentrations of the three mRNA and repressorproteins, and $\gamma>0$ denotes the ratio of the protein decay rate to mRNA decay rate. By taking into account 

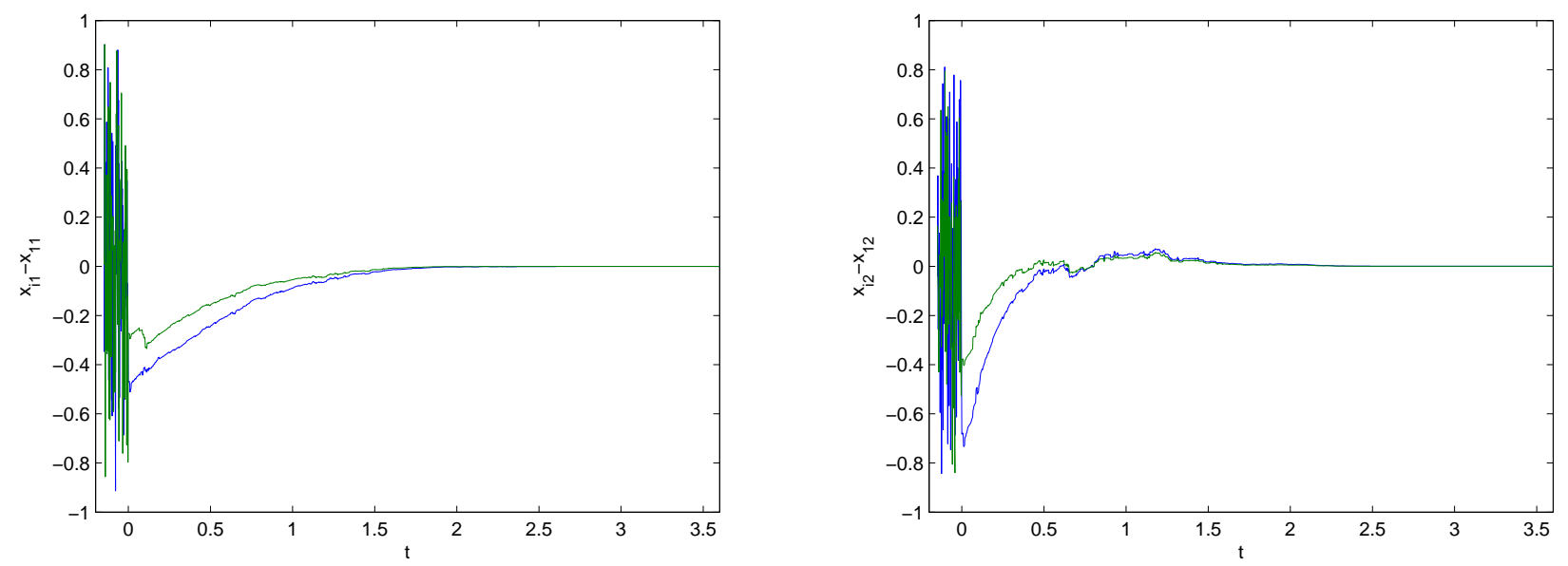

Fig. 5. Synchronization error of $x_{i 1}(t)-x_{11}(i=2,3)$ for Fig. 6. Synchronization error of $x_{i 2}(t)-x_{12}(i=2,3)$ for model (44) model (44)

the transcriptional time delay and selecting a set of biologically plausible parameters, the above equations can be rewritten into the following vector form:

$$
d y(t)=[A y(t)+D y(t-\tau(t))+B f(y(t))] d t, \quad t>0
$$

where $y(t)=\left(y_{1}(t), y_{2}(t), \ldots, y_{6}(t)\right)^{T} \in \mathbb{R}^{6} ; A=\operatorname{diag}(-3,-3,-3,-2.5,-2.5,-2.5)$; the time-varying delay $\tau(t)=\frac{1}{4}(1+\sin t)$, i.e., $\tau_{M}=0.5, \mu=0.25$; the nonlinear function $f(y(t))=\left(f_{1}\left(y_{1}(t)\right), f_{2}\left(y_{2}(t)\right), \ldots, f_{6}\left(y_{6}(t)\right)\right)^{T}$ with $f_{i}\left(y_{i}(t)\right)=1 /\left(1+y_{i}^{2}(t)\right)(i=1,2, \ldots, 6)$, obviously, the Assumption 1 is satisfied with $X_{1}=\operatorname{diag}(-0.6495$, $-0.6495,-0.6495,-0.6495,-0.6495,-0.6495)$ and $X_{2}=0$;

$$
D=\left[\begin{array}{cccccc}
0 & 0 & 0 & 0 & 0 & 0 \\
0 & 0 & 0 & 0 & 0 & 0 \\
0 & 0 & 0 & 0 & 0 & 0 \\
0.8 & 0 & 0 & 0 & 0 & 0 \\
0 & 0.8 & 0 & 0 & 0 & 0 \\
0 & 0 & 0.8 & 0 & 0 & 0
\end{array}\right], \quad B=\left[\begin{array}{cccccc}
0 & 0 & 0 & 0 & 0 & 2.5 \\
0 & 0 & 0 & 2.5 & 0 & 0 \\
0 & 0 & 0 & 0 & 2.5 & 0 \\
0 & 0 & 0 & 0 & 0 & 0 \\
0 & 0 & 0 & 0 & 0 & 0 \\
0 & 0 & 0 & 0 & 0 & 0
\end{array}\right]
$$

Now consider a coupled complex network consisting of three identical models (45), the state equations of the entire array are

$$
d x_{i}(t)=\left[A x_{i}(t)+D x_{i}(t-\tau(t))+B f\left(x_{i}(t)\right)+\sum_{j=1}^{3} G_{i j}^{(1)} \Gamma_{1} x_{j}(t)+\sum_{j=1}^{3} G_{i j}^{(2)} \Gamma_{2} x_{j}(t-\tau(t))\right] d t
$$

where $x_{i}(t)=\left(x_{i 1}(t), x_{i 2}(t), \ldots, x_{i 6}(t)\right)^{T}(i=1,2,3)$ is the state vector of the $i$ th subsystem. Choose the coupling matrices $G^{(1)}, G^{(2)}$ and the linking matrices $\Gamma_{1}, \Gamma_{2}$ as

$$
G^{(1)}=\left[\begin{array}{ccc}
-0.2 & 0.1 & 0.1 \\
0.1 & -0.2 & 0.1 \\
0.1 & 0.1 & -0.2
\end{array}\right], \quad \Gamma_{1}=\left[\begin{array}{cccccc}
0.8055 & 0.4899 & 0.0596 & 0.8181 & 0.9730 & 0.0835 \\
0.5767 & 0.1679 & 0.6820 & 0.8175 & 0.6490 & 0.1332 \\
0.1829 & 0.9787 & 0.0424 & 0.7224 & 0.8003 & 0.1734 \\
0.2399 & 0.7127 & 0.0714 & 0.1499 & 0.4538 & 0.3909 \\
0.8865 & 0.5005 & 0.5216 & 0.6596 & 0.4324 & 0.8314 \\
0.0287 & 0.4711 & 0.0967 & 0.5186 & 0.8253 & 0.8034
\end{array}\right]
$$




$$
G^{(2)}=\left[\begin{array}{ccc}
-0.3 & 0.1 & 0.2 \\
0.1 & -0.2 & 0.1 \\
0.2 & 0.1 & -0.3
\end{array}\right], \quad \Gamma_{2}=\left[\begin{array}{cccccc}
0.0605 & 0.2920 & 0.3724 & 0.0527 & 0.4177 & 0.6981 \\
0.3993 & 0.4317 & 0.1981 & 0.7379 & 0.9831 & 0.6665 \\
0.5269 & 0.0155 & 0.4897 & 0.2691 & 0.3015 & 0.1781 \\
0.4168 & 0.9841 & 0.3395 & 0.4228 & 0.7011 & 0.1280 \\
0.6569 & 0.1672 & 0.9516 & 0.5479 & 0.6663 & 0.9991 \\
0.6280 & 0.1062 & 0.9203 & 0.9427 & 0.5391 & 0.1711
\end{array}\right]
$$

By using the Matlab LMI Toolbox, LMIs (38) can be solved with the feasible solutions as follows (for space consideration, only some of them are listed):

$$
\begin{aligned}
& P=\left[\begin{array}{cccccc}
47.4489 & -3.7570 & -1.1316 & 0.1900 & -2.5717 & -1.1133 \\
-3.7570 & 46.9208 & -3.2133 & -4.9917 & -2.1384 & -3.8429 \\
-1.1316 & -3.2133 & 46.1916 & -1.9864 & -2.5593 & 1.2069 \\
0.1900 & -4.9917 & -1.9864 & 70.2065 & 1.5434 & -2.9769 \\
-2.5717 & -2.1384 & -2.5593 & 1.5434 & 64.9746 & -5.6841 \\
-1.1133 & -3.8429 & 1.2069 & -2.9769 & -5.6841 & 69.2279
\end{array}\right] \\
& Q_{1}=\left[\begin{array}{cccccc}
111.8939 & -10.4443 & -3.0019 & 7.1653 & 10.7947 & 24.0458 \\
-10.4443 & 98.6225 & -0.5504 & 20.4771 & 1.9922 & -13.7916 \\
-3.0019 & -0.5504 & 96.4250 & 1.1320 & 25.9715 & 7.7806 \\
7.1653 & 20.4771 & 1.1320 & 134.5675 & 26.3377 & 8.1183 \\
10.7947 & 1.9922 & 25.9715 & 26.3377 & 122.3597 & 10.7605 \\
24.0458 & -13.7916 & 7.7806 & 8.1183 & 10.7605 & 158.5158
\end{array}\right] \\
& T_{1}=\left[\begin{array}{cccccc}
4.3514 & 0.2416 & 0.7972 & -2.7124 & -0.1404 & 1.3519 \\
0.5996 & 4.8981 & -0.5755 & -0.6869 & 1.8925 & 0.8451 \\
1.1770 & -0.2907 & 5.7473 & 0.6206 & -2.6226 & 0.2936 \\
-3.7692 & -0.0994 & 1.0228 & -1.7906 & 5.6290 & 1.1377 \\
0.9733 & -4.1312 & 0.4865 & 4.7749 & -1.6056 & 8.3088 \\
-0.8207 & -0.6842 & -2.1438 & 8.4909 & 5.1912 & -3.7950
\end{array}\right]
\end{aligned}
$$

and $\beta=108.8948$. Therefore, according to Corollary 2, the array of the three coupled biological networks (46) can achieve global synchronization.

\section{Conclusions}

In this paper, we have dealt with the synchronization problem for a general class of coupled complex networks. The system under study are coupled by $N$ identical subsystems, each of them has uncertain parameters and stochastic disturbances, and they are linearly stochastically coupled which may reflect a more realistic situation in practice. By employing the Lyapunov functional method combined with the matrix inequalities and by using the properties of Kronecker product, several sufficient conditions have been obtained which ensure the coupled complex network to be globally robustly synchronized in the mean square. The criteria acquired in this paper are dependent on the upper bound of the time-varying delay which may be less conservative than the delay-independent ones, and the derivative of the time-varying delay is not required to be less than 1. Furthermore, these criteria can be easily verified by using the standard numerical software. In the end of the paper, two examples have been given with simulations to demonstrate the effective of our results. 


\section{REFERENCES}

[1] S. Boyd, L.E. Ghaoui, E. Feron, V. Balakrishnan, Linear matrix inequalities in system and control theory, Philadelphia: SIAM, 1994.

[2] N. Burić, K. Todorović, N. Vasović, Synchronization of noisy delayed feedback systems with delayed coupling, Phys. Rev. E, vol. 75, 026209, 2007.

[3] J. Cao, P. Li, W.W. Wang, Global synchronization in arrays of delayed neural networks with constant and delayed coupling, Phys. Lett. A, vol. 353, no. 4, pp. 318-325, 2006.

[4] J. Cao, Z. Wang, Y. Sun, Synchronization in an array of linearly stochastically coupled neural networks with time delays, Physica A, vol. 385, no. 2, pp. 718-728, 2007.

[5] G. Chen, J. Zhou, Z.R. Liu, Global synchronization of coupled delayed neural networks and applications to chaotic CNN models, Int. J. Bifurc. Chaos, vol. 14, no. 7, pp. 2229-2240, 2004.

[6] J.L. Chen, X.H. Chen, Special matrices, Tsinghua University Press, China, 2001.

[7] C.J. Cheng, T.L. Liao, J.J. Yan, C.C. Hwang, Exponential synchronization of a class of neural networks with time-varying delays, IEEE Trans. Systems, Man and Cybernetics - Part B, vol. 36, no. 1, pp. 209-215, 2006.

[8] M.B. Elowitz, S. Leibler, A synthetic oscillatory network of transcriptional regulators, Nature, vol. 403, pp. 335-338, 2000.

[9] A. Friedman, Stochastic differential equations and their applications, Academic Press, New York, 1976.

[10] H. Gao, J. Lam, G. Chen, New criteria for synchronization stability of general complex dynamical networks with coupling delays, Phys. Lett. A, vol. 360, pp. 263-273, 2006.

[11] H. Gao, T. Chen, New results on stability of discrete-time systems with time-varying state delay, IEEE Tran. Autom. Control, vol. 52, no. 2, pp. 328-334, 2007.

[12] K.Q. Gu, V.L. Kharitonov, J. Chen, Stability of time-delay systems, Boston: Birkhauser, 2003.

[13] Y.Y. Hou, T.L. Liao, J.J. Yan, Stability analysis of Takagi-Sugeno fuzzy cellular neural networks with time-varying delays, IEEE Trans. Systems, Man and Cybernetics - Part B, vol. 37, no. 3, pp. 720-726, 2007.

[14] X. Hu, J. Wang, Design of general projection neural networks for solving monotone linear variational inequalities and linear and quadratic optimization problems, IEEE Trans. Systems, Man and Cybernetics - Part B, vol. 37, no. 5, pp. 1414-1421, 2007.

[15] J. Jost, M. Joy, Special properties and synchronization in coupled map lattices, Phys. Rev. E, vol. 65, $061201,2002$.

[16] H.K. Khalil, Nonlinear systems, Upper Saddle River, NJ:Prentice-Hall, 1996.

[17] C.G. Li, G. Chen, Synchronization in general complex dynamical networks with coupling delays, Physica A, vol. 343, pp. 263-278, 2004.

[18] Z. Li, G. Chen, Global synchronization and asymptotic stability of complex dynamical networks, IEEE Trans. Circuits Syst.-II, vol. 53, no. 1, pp. 28-33, 2006.

[19] C. Li, L. Chen, K. Aihara, Stability of genetic networks with SUM regulatory logic: Lur'e system and LMI approach, IEEE Transactions on Circuits Systems-I, vol. 53, no. 11, pp. 2451-2458, 2006.

[20] J. Liang, Z. Wang, Y. Liu and X. Liu, Global synchronization control of general delayed discrete-time networks with stochastic coupling and disturbances, IEEE Trans. Systems, Man, and Cybernetics - Part B, in press.

[21] Y. Liu, Z. Wang and X. Liu, Robust stability of discrete-time stochastic neural networks with time-varying delays, Neurocomputing, vol. 71, no. 4-6, pp. 823-833, 2008.

[22] J.Q. Lu, J. Cao, Synchronization-based approach for parameters identification in delayed chaotic neural networks, Physica A, vol. 382, pp. 672-682, 2007.

[23] J.Q. Lu, D.W.C. Ho, M. Liu, Globally exponential synchronization in an array of asymmetric coupled neural networks, Phys. Lett. A, vol. 369, no. 5-6, pp. 444-451, 2007.

[24] W.L. Lu, T.P. Chen, New approach to synchronization analysis of linearly coupled ordinary differential systems, Physica D, vol. 213, pp. 214-230, 2006.

[25] W.L. Lu, T.P. Chen, G. Chen, Synchronization analysis of linearly coupled systems described by differential equations with a coupling delay, Physica D, vol. 221, no. 2, pp. 118-134, 2006

[26] J.H. Lü, G. Chen, A time-varying complex dynamical network model and its controlled synchronization criteria, IEEE Trans. Autom. Control, vol. 50, pp. 841-846, 2005.

[27] R. Palm, Synchronization of decentralized multiple-model systems by market-based optimization, IEEE Trans. Systems, Man and Cybernetics - Part B, vol. 34, no. 1, pp. 665-672, 2004.

[28] L.M. Pecora, T.L. Carroll, Synchronization in chaotic systems, Phys. Rev. Lett., vol. 64, no. 8, pp. 821-824, 1990.

[29] V. Perez-Munuzuri, V. Perez-Villar, L.O. Chua, Autowaves for image processing on a two-dimensional CNN array of excitable nonlinear circuits: flat and Wrinkled labyrinths, IEEE Trans. Circuits Syst.-I, vol. 40, pp. 174-181, 1993.

[30] Z. Schuss, Theory and applications of stochastic differential equations, Wiley, New York, 1980.

[31] S. Senan, S. Arik, Global robust stability of bidirectional associative memory neural networks with multiple time delays, IEEE Trans. Systems, Man and Cybernetics - Part B, vol. 37, no. 5, pp. 1375-1381, 2007. 
[32] Q. Song and J. Cao, Impulsive effects on stability of fuzzy Cohen-Grossberg neural networks with time-varying delays, IEEE Trans. Systems, Man and Cybernetics - Part B, vol. 37, no. 3, pp. 733-741, 2007.

[33] X.F. Wang, G. Chen, Synchronization in small-world dynamical networks, Int. J. Bifurc. Chaos, vol. 12, no. 1, pp. 187-192, 2002.

[34] Z. Wang, Y. Liu, M. Li and X. Liu, Stability analysis for stochastic Cohen-Grossberg neural networks with mixed time delays, IEEE Trans. Neural Networks, vol. 17, no. 3, pp. 814-820, 2006.

[35] Z. Wang, F. Yang, D. W. C. Ho and X. Liu, Robust $H_{\infty}$ control for networked systems with random packet losses, IEEE Trans. Syst. Man, Cybern.-B, vol. 37, no. 4, pp. 916-924, 2007.

[36] Z. Wang, J. Lam, L. Ma, Y. Bo and Z. Guo, Variance-constrained dissipative observer-based control for a class of nonlinear stochastic systems with degraded measurements, Journal of Mathematical Analysis and Applications, Vol. 377, No. 2, May 2011, pp. 645-658.

[37] Z. Wang, D. W. C. Ho, H. Dong and H. Gao, Robust $H_{\infty}$ finite-horizon control for a class of stochastic nonlinear time-varying systems subject to sensor and actuator saturations, IEEE Transactions on Automatic Control, Vol. 55, No. 7, Jul. 2010, pp. 1716-1722.

[38] Z. Wang, Y. Liu, G. Wei and X. Liu, A note on control of a class of discrete-time stochastic systems with distributed delays and nonlinear disturbances, Automatica, Vol. 46, No. 3, Mar. 2010, pp. 543-548.

[39] Z. Wang, Y. Liu and X. Liu, Exponential stabilization of a class of stochastic system with Markovian jump parameters and mode-dependent mixed time-delays, IEEE Transactions on Automatic Control, Vol. 55, No. 7, Jul. 2010 , pp. $1656-1662$.

[40] Z. Wang, Y. Wang and Y. Liu, Global synchronization for discrete-time stochastic complex networks with randomly occurred nonlinearities and mixed time-delays, IEEE Transactions on Neural Networks, Vol. 21, No. 1, Jan. 2010 , pp. 11-25.

[41] C.W. Wu, L.O. Chua, Synchronization in an array of linearly coupled dynamical systems, IEEE Trans. Circuits Syst.-I, vol. 42, no. 8, pp. 430-447, 1995.

[42] C.W. Wu, Synchronization in arrays of coupled nonlinear systems with delay and nonreciprocal time-varying coupling, IEEE Trans. Circuits Syst.-II, vol. 52, no. 5, pp. 282-286, 2005.

[43] L. Xie, Output feedback $H_{\infty}$ control of systems with parameter uncertainty, Int. J. Control, vol. 63, pp. 741-750, 1996.

[44] K. Yuan, J. Cao, H.-X. Li, Robust stability of switched Cohen-Grossberg neural networks with mixed time-varying delays, IEEE Trans. Systems, Man and Cybernetics - Part B, vol. 36, no. 6, pp. 1356-1363, Dec. 2006.

[45] W.W. Yu, J. Cao, Synchronization control of stochastic delayed neural networks, Physica A, vol. 373, pp. 252-260, 2007.

[46] A. Zheleznyak, L.O. Chua, Coexistence of low- and high-dimensional spatio-temporal chaos in a chain of dissipatively coupled Chua's circuits, Int. J. Bifurc. Chaos, vol. 4, no. 3, pp. 639-674, 1994.

[47] J. Zhou, T.P. Chen, Synchronization in general cpmplex delayed dyanmical networks, IEEE Trans. Circuits Syst.-I, vol. 43, no. 3, pp. 733-744, 2006 